\title{
Gestión de la Educación basada en competencias: elementos para su interpretación en el contexto de la Administración de la Educación
}

\author{
Educational management based in competences: elements for its \\ interpretation in the context of administration of the education
}

\author{
Pedro Venegas Jiménez \\ Universidad de Costa Rica \\ pvenegas@ice.co.cr
}

Recibido: 08 noviembre 2010 Aceptado: 09 noviembre 2010 Corregido: 28 junio 2011

\section{Resumen}

El nuevo siglo trae consigo cambios vertiginosos que se extienden a la realidad en los centros educativos, los cuales han tenido que variar sus formas de gestión como resultado de los nuevos retos y nuevas competencias que presenta la sociedad actual. Estudiar las competencias en gestión educativa permite comprender el rol del administrador de la educación, importante para el mejoramiento de la educación nacional en un contexto mundial. Todo ello, conduce a la necesidad de indagar elementos de un perfil del administrador de la educación basado en competencias, desde la perspectiva de su gestión profesional.

El escenario de aprendizaje más inmediato para el desarrollo del gestor de la educación es el centro educativo, mediante un conjunto de elementos propios de la teoría contemporánea en este campo del saber. El autor se sustenta en diversas publicaciones producto de su experiencia profesional en la formación de administradores de la educación y en la participación en proyectos basados en competencias.

Palabras Clave: educación, gestión, gestión educativa, competencias, administrador de la educación, gestión por competencias. 


\begin{abstract}
The new century brings rapid changes that extend to the reality in schools, which have had to change their ways of management as a result of new challenges and responsibilities presented by the society. Is very important to the improvement of national education in a global context, to study the education management skills to understand the role of educational administrator. This leads to the need to investigate elements of a profile manager competency-based education from the perspective of professional management. The most immediate learning setting for the development manager of education is the school, through a set of elements of the contemporary theory of knowledge in this field. The author is supported by several publications product of professional experience in the training of educational administrators and participating in projects based on skills.
\end{abstract}

Keywords: education, management, education management, skills, competency management, education administrator.

\title{
1. Presentación
}

Los centros educativos de inicio de un nuevo siglo no pueden seguir siendo los mismos del siglo pasado. La época actual presenta nuevos retos ante la necesidad de alcanzar nuevas competencias propias del mundo de cambios vertiginosos en el que se vive, como respuesta a las necesidades de la sociedad y del centro educativo el día de hoy. Es fundamental que se analicen entonces, las formas de gestión en los centros educativos en un contexto de complejidad, caos, multiculturalidad y diversidad (Sagastizabal, Perlo, Pivetta y San Martín, 2006).

La educación que se vive en estos años requiere ser una educación para la vida, por lo que se desarrolla, precisamente, a lo largo de ella, como parte de una sociedad educativa en la que se ofrecen múltiples posibilidades de aprender. Incluso, es fundamental, en esta sociedad del siglo XXI, referir a Delors, Mufti, Amagi, Carneiro, Chung, Geremek, y Nanzhao (1996), cuando menciona la necesidad de aprender a aprender.

Así, los sistemas educativos deben responder a los múltiples retos que les lanza la sociedad de la información; una sociedad globalizada que demanda la renovación de procesos, ideas y actitudes ante la necesidad impostergable de una gestión de la educación 
diferente para una educación también diferente. Esto requiere, de antemano, enriquecer la gestión educativa con la puesta en práctica de los cuatro pilares de la educación: aprender a conocer, aprender a hacer, aprender a ser y aprender a convivir (Delors et al., 1996).

En este artículo se incluyen elementos de carácter general para el estudio de competencias en la gestión educativa. En este sentido, se trata de un artículo introductorio, orientado hacia la reflexión para el análisis e interpretación de los diversos referentes considerados en cada uno de los apartados. Cada componente representa, en sí mismo, áreas de estudio para el desarrollo de competencias específicas. Se trata de un campo en el que diversos autores han dado aportes significativos, especialmente en estos años iniciales del siglo XXI.

El estudio, la vivencia y el intercambio de experiencias, mediante los diversos medios propios de la época, permite el conocimiento de este mundo de múltiples relaciones y alcances, sociedad de complejidad y diversidad, con pluralidad de situaciones en cada rincón del planeta, cada una de las cuales permite la comprensión de la propia realidad y, en este caso en particular, la realidad de los centros educativos. El desarrollo de competencias y su gestión será el recurso para el acercamiento a la comprensión de cada realidad. El rol del administrador de la educación, concebido como gestor, es clave en este entorno.

Este artículo está constituido por tres partes fundamentales: concepto de gestión desde la perspectiva educativa, referentes de competencias en el contexto de la administración de la educación y presentación de algunas ideas para un perfil de gestión en administración de la educación.

\section{Gestión en educación}

\subsection{Hacia el concepto de gestión}

La gestión escolar, como filosofía de gestión de las organizaciones, constituye un cambio de paradigma que incluye valores, principios y procedimientos como estrategia de 
progreso por excelencia para las próximas décadas. En este contexto, es preciso caracterizar el concepto de gestión.

Interpretando a Casassus (2000), gestión es un concepto más genérico que administración, ya que va mucho más allá de la ejecución de instrucciones, de manera que gestión connota tanto las acciones de planificar como las de administrar. En el contexto escolar, el término fue utilizado de forma reducida a la ejecución de planes o a las actividades relacionadas exclusivamente con el manejo de los recursos materiales humanos y primordialmente, financieros, sin que tuviera una injerencia en los ámbitos pedagógicos y curriculares.

"La gestión, como concepto amplio que es, se relaciona con otras palabras que comparten su raíz latina, tales como gesta, gestación, gesticulación, gestual y gerencia, y las involucra a todas dentro de sí” (Betancourt, 2002, p. 27). De esta manera, la gestión, en su acepción como gesta busca una hazaña, una proeza, el logro de algo diferente a lo tradicional. En educación, precisamente, buscamos la gestión para superar los enfoques arcaicos y tener acceso a una educación de calidad. De ahí, que en la gestión escolar, el trabajo del administrador de la educación sea definido, recientemente, como lo indica Blejmar (referido por Romero, 2009), “hacer que las cosas sucedan” (p.12).

Gestionar significa, por tanto, “definir objetivos y medir resultados, administrar recursos, buscar la eficiencia. Se trata de un quehacer dirigido a garantizar previsibilidad, racionalidad y responsabilidad por los resultados” (Romero 2009, p. 11). Por esto, según la misma autora, "mejorar la gestión escolar significa empezar a pensarla en el contexto de la mejora escolar” (Romero, 2009, p. 13).

De acuerdo con Ibarrola (citada por Pozner, 2000), la palabra gestión alude a un nuevo concepto que tiene que ver con la manera como se administran, en el sentido más notable y más completo, los muy distintos insumos que intervienen en los complejos educativos. Con esto se quiere decir, según la misma autora, que la gestión conduce a una "mejor y más eficiente organización educativa parta alcanzar los nuevos objetivos que nos impone una sociedad que vive cambios tan profundos” (Ibarrola, referida por Pozner, 2000, p. 25). 
También la gestión se concibe como un proceso de aprendizaje de la adecuada relación entre estructura, estrategia, sistemas, estilo, capacidades, gente y objetivos superiores, tanto hacia el interior de la organización como hacia el entorno (Pérez, 1999). Así, gestión es un proceso de aprendizaje orientado a la supervivencia de una organización mediante una articulación constante con el entorno o contexto. En otras palabras, el concepto de gestión lleva implícito el concepto de aprendizaje, generación de valores, visión compartida, interacciones, representaciones mentales. Se establece, entonces, una relación estrecha entre educación y gestión.

Bajo este nuevo concepto, interesa mucho, como parte de la gestión, el comportamiento organizacional, el compromiso, la satisfacción en el trabajo. Con ello, es fundamental el estudio y énfasis en el liderazgo, relaciones interpersonales, comunicación, trabajo en equipo en los centros educativos. Estos elementos (competencias) constituyen los pilares del nuevo enfoque de gestión en educación, no limitada a la administración de recursos humanos, sino enfocada especialmente hacia el desarrollo de competencias, entre ellas el liderazgo pedagógico. Así, se concibe que la gestión implica trabajar con personas, no con máquinas.

El concepto de gestión se aleja de una visión restringida, propia de la orientación burocrático-administrativa, para adquirir un significado más global, comprensivo de la gestión de los recursos, de las personas, de los procesos y de los resultados, elementos que, en interacción recíproca, se dan cita en toda organización. Se entiende entonces, la gestión escolar, como un conjunto de acciones articuladas entre sí, que posibilitan la consecución de la intencionalidad de la institución, como aquel proceso que articula las múltiples instancias de la vida escolar con una sola dirección, la de lograr aprendizajes significativos en los alumnos. Gestionar, implica enlazar todo aquello que acontece todos los días, es hacer posible el logro del propósito de la institución, por medio de la articulación de las acciones que permiten la finalidad educativa de la institución (Lavín, 1998).

\subsection{Algunos elementos básicos de la educación y su gestión}


Uno de los factores clave para el desarrollo de una sociedad es la educación que se vive en ella y su administración en todos los niveles, desde el despacho de un Ministro hasta la más alejada de las escuelas.

Es en cada aula y en cada centro educativo donde todo intento de mejoramiento educativo se hace realidad. De ahí, que cada centro educativo también debe tener la oportunidad y derecho de dar sus propios aportes, de elaborar y desarrollar sus propios proyectos, de tratar de tener su propia identidad, sustentados en una cultura propia, acorde con sus auténticas necesidades (Venegas, 2004).

Todos los educadores (docentes, directores, asesores, supervisores, administrativos y otros), estudiantes y padres de familia tienen un gran potencial humano y profesional, el cual no ha sido bien desarrollado; han faltado oportunidades para su mejor aprovechamiento profesional.

¿Donde, si no en el ámbito educativo, se encuentra el escenario más favorable para brindar oportunidades que permitan pensar, crear, ser innovadores, dar aportes significativos, ser mejores personas y desarrollar todo el talento humano en cada contexto en que nos desenvolvemos?

Bien lo refiere Pozner (2000) cuando afirma que “el problema principal del funcionamiento de los sistemas no ha sido tanto algo que haya hecho -controlar en demasía de manera centralizada impidiendo la libertad y la autonomía-, sino algo que no han hecho: crear capacidad profesional e institucional en todos los rincones del sistema” (p.20).

Hay que tener confianza en cada docente, en cada estudiante, en cada administrador gestor de la educación. Creen en ellos significa confiar en sus capacidades, las cuales muchas veces han sido ignoradas o al menos subestimadas, siendo que todas las personas cuentan con inteligencias en diversos campos.

De acuerdo con UNESCO (2004), la gestión educativa es un saber capaz de ligar conocimientos, acción ética y administración en procesos que tienden al mejoramiento continuo de las prácticas educativas, a la exploración y explotación de todas las posibilidades y la innovación permanente como proceso sistemático. En este contexto, el verdadero respeto al profesional en educación consiste en hacerlo partícipe en el desarrollo 
de su propia profesión. La innovación y la creatividad, así como el respeto y estímulo por lo que realizan en el desempeño de su cargo, deben ser factores implícitos en su gestión educativa que requieren ser fomentados y tomados en cuenta para el desarrollo de la educación, que les permita, a su vez, desarrollar una gestión profesional diferente, rica en oportunidades y acciones curriculares en un marco educativo que se sustenta en competencias.

Desde esta perspectiva, todos los miembros tienen oportunidad de participar, de manera que la planificación y la toma de decisiones pertinentes se tornan colectivas, como parte de un trabajo en equipo. Al interior de los centros escolares, la participación, como señala Antúnez (2000), puede orientarse a finalidades educativas, de gestoría, de relación con el currículo, de control social y de interiorización del proyecto educativo de centro. La orientación de estas organizaciones en la gestión educativa es a largo plazo, por lo que se piensa en una persona que labore en ella durante toda su vida, ya que funcionario e institución se necesitan mutuamente.

En estas organizaciones, las promociones obedecen a criterios múltiples, no fundamentadas únicamente en el rendimiento o productividad individual, sino básicamente en su participación dentro de un equipo de trabajo, su antigüedad, sus relaciones con los demás, su creatividad, su espíritu de innovación y propuestas de solución y de mejoramiento ante las diversas situaciones que enfrenta diariamente (Venegas, 2000). Se quiere al funcionario para siempre, con participación y conocimiento de lo que ahí se hace; se aspira a un funcionario con iniciativa, para lo cual cuenta con el apoyo de todos y es concebido como persona capaz, que está aprendiendo constantemente y que también, enseña permanentemente.

Dado que todos los individuos tienen la oportunidad de participar, proponer y tomar las decisiones que corresponden a su ámbito, aquellas organizaciones que desarrollan su gestión basada en competencias, en el contexto de las corrientes de inicio del siglo XXI, se orientan hacia una cultura y filosofía organizacional bien definidas y conocidas por todos los miembros (Venegas, 2011). 
De esta manera, la comunicación ha de darse de manera directa entre director y docentes, entre docentes y estudiantes, entre docentes y padres de familia y, de igual manera, entre todos y cada uno de los diferentes integrantes asociados con el quehacer de la gestión educativa. Esta comunicación debe ser fluida y debe ser normal y rutinario que la iniciativa de la comunicación se lleve a cabo desde la base hasta la cúspide.

Ya no se piensa en aquella pirámide de jerarquía tradicional, sino en una pirámide invertida para los procesos de comunicación (Venegas, 2008). Para que esta comunicación sea directa debe tenerse la confianza de los demás y sobretodo, en la persona que ejerza la dirección en el centro educativo, que actúa concibiendo a los demás como personas con sentimientos, necesidades físicas y emocionales, que van a estar en la organización durante muchos años más, capaces de hacer muchas cosas, y hacerlas bien. Para Venegas (2004), debe haber un sentimiento arraigado en el interior de cada uno de necesitarse mutuamente como personas, como seres humanos y como profesionales de la educación.

Al respecto, refieren Elizondo y Mora (2011) que la comunicación ocupa un lugar primordial, ya que colabora en la información y toma de decisiones entre los individuos en las organizaciones. Además, los autores refieren a que "la interacción con los alumnos, colegas, administrativos, padres de familia y personas de las comunidad es parte de la vida cotidiana del docente y le permite expresar formas de pensar y ser” (Elizondo y Mora, 2011, p. 42).

Se trata, pues, de una gestión con énfasis en las relaciones informales. Se necesita el contacto permanente con los demás. Este contacto se lleva a cabo en el campo, en los pasillos, en la tertulia durante el café, en el intercambio profesional sustentado en el respeto. No se trata, pues, de un liderazgo de escritorio, de ceremonia, de relación jefesubordinado.

Estos referentes conceptuales permiten hacer énfasis en los siguientes elementos de la gestión educativa:

- La gestión escolar conjuga la teoría con la práctica. Todo lo que se hace en un centro educativo obedece a la formación y forma de ser de todos sus miembros, 
representados por un director o directora que, dada su autoridad formal, ejerce influencia en el quehacer del centro educativo. No obstante, la noción de gestión escolar no se reduce a la función del director, sino que intenta poner dicha función en relación con el trabajo colegiado del personal y hacerla partícipe de las relaciones que se establecen entre los diferentes actores de la comunidad educativa (Antúnez, 2000; Lavín, 1998; Luque, 2003; Pozner 2000; Romero, 2009).

- “La gestión escolar, centrada en hacer de la escuela un proyecto y gestar su mejora, es un asunto complejo y multidimensional” (Romero, 2009, p.14). Esto da a entender con claridad el concepto de gestión para lograr mejores resultados en los centros educativos.

- La gestión escolar debe crear condiciones favorables para que el estudiante aprenda a ser (ser persona, ser solidario, ser conciliador, ser compañero), aprenda a vivir con los demás, aprenda a conocer, a aprender y a emprender. Esto también conduce a que los miembros de la comunidad educativa pretendan autonomía en busca de decisiones propias con responsabilidad, con el propósito de tener una vida feliz como parte de una comunidad y aprender, permanentemente, con todos los miembros.

Parte sustancial de este concepto es la gestión del currículo, con el fin de lograr una buena gestión pedagógica, con involucramiento permanente por parte de todos los actores del proceso educativo. Al tenor, como se ha referido previamente, los procesos de enseñanza y aprendizaje, constituyen, en gran parte, la razón de ser de este tipo de organizaciones, y no pueden aislarse del resto de los procesos que se llevan a cabo en el centro educativo (Romero, 2009). Así, por ejemplo, para analizar e interpretar el clima escolar hay que concebirlo como un conjunto de factores tales como la labor de cada docente, el estilo de dirección, relaciones entre colegas, aspectos propios de la infraestructura, procedimientos internos, entre otros, todos relacionados con el desarrollo de los procesos de enseñanza y aprendizaje y la gestión curricular. 
La gestión de la educación adquiere sentido cuando se hace referencia al enfoque por competencias. Esta relación es la que se trata de especificar en el siguiente apartado.

\section{Competencias en administración y gestión de la educación}

En la sociedad actual, reconocida como sociedad del conocimiento y la información, como parte de un mundo globalizado, es ineludible vincularse al ámbito de las competencias. Para tal efecto, es necesario referir al significado y consecuencias del paradigma de la gestión de la educación basada en competencias, para lo que se presentan los propósitos de la educación en este sentido. Esto lleva a la identificación de competencias en la gestión escolar y a una propuesta taxonómica básica de competencias orientadas al ámbito de la administración de la educación. Finalmente, se aportan competencias que debe poseer el gestor en administración de la educación en términos de una gestión educativa basada en competencias (perfil), en su condición de profesional que desarrolla su labor en el contexto del siglo XXI, con todas las particularidades propias de esta época.

\subsection{Propósitos de educación por competencias}

El concepto de competencia se establece desde el siglo V a.C., en relación con las palabras de Confucio que llevan a oir y olvidar, ver y recordar, hacer y comprender (Páez, 2009).

El término competencia proviene del latín competeré, palabra que se relaciona con el aspirar e ir al encuentro de algo. Luego derivó en competer: incumbir, pertenecer, estar investido de autoridad para ciertos asuntos. Hoy se utiliza el término competente: quien se desenvuelve con eficacia.

Por competencia, entonces, se entiende aquella capacidad de responder a demandas complejas y llevar a cabo tareas diversas de forma adecuada. O bien, como el conjunto de conocimientos, actitudes y destrezas necesarias para desempeñar una ocupación dada. En 
este sentido la competencia implica la capacidad de generar aplicaciones o soluciones adaptadas a la situación, movilizando los propios recursos y regulando el proceso hasta lograr la meta pretendida (Argüelles, 1996).

\subsection{Competencias en la gestión de la educación}

En un centro educativo cuya gestión se basa en competencias, se estrecha la relación entre escuela y comunidad, se disfruta de la autonomía necesaria y se proporcionan todas las condiciones para que se aplique una gestión y planificación del currículo con carácter gerencial y de liderazgo (OEA-MEP, 2006). En este contexto, la gestión se sustenta en el ser humano como elemento indispensable dentro de la organización, con participación activa como miembro de la organización, desde la planificación institucional hasta la toma de decisiones correspondiente en cada nivel.

De manera consecuente, la gestión educativa de este inicio de siglo debe llevarse a cabo con una orientación a largo plazo, dentro de una concepción integral. Esta integralidad, de acuerdo con Elizondo y Mora (2011) debe orientarse hacia el desarrollo de competencias para el mejoramiento del proceso educativo, ya que este

Es sin duda la principal función y responsabilidad del docente, pero hoy en día es además casi una exigencia el desarrollar las competencias de distintos ámbitos que la institución requiera, todo esto para tener como resultado un proceso de enseñanza- aprendizaje de calidad que responda a los requerimientos de la sociedad actual. (p. 9)

En relación con el desempeño profesional de los administradores de la educación, se puede afirmar que, en su calidad de gestor educativo, es competente si está capacitado para enfrentar las demandas del medio en el que se desenvuelve y realiza tareas en forma exitosa. 
Se es competente cuando existe la capaz de resolver con entendimiento situaciones y problemas, haciendo valer sus pensamientos, sus actuaciones y demostrando que su preparación es mayor que la del resto.

Estas definiciones permiten derivar que las competencias:

- Son características permanentes de la persona

- Se ponen de manifiesto cuando se ejecuta una tarea o se realiza un trabajo

- Están relacionadas con la ejecución exitosa en una actividad, laboral o de otra índole

- Tienen una relación causal con el rendimiento laboral, es decir, no están solamente asociadas con el éxito, sino que se asume que realmente lo causan.

- Pueden ser generalizables a más de una actividad (Venegas, 2011).

En este concepto, se manifiesta la gestión de la educación sustentada en el enfoque por competencias.

Al referir una conceptualización diferente para llevar a cabo el proceso de gestión en centros educativos, acorde con la época actual, caracterizada por constantes y súbitos cambios, se está ubicado en el contexto de un pensamiento estratégico que caracteriza a lo que, en estos días, se denomina organizaciones inteligentes (Martin, 2001). Esta gestión se manifiesta en el enfoque por competencias y en los fundamentos del Proyecto Estratégico de Centro Educativo (PECE), de acuerdo con Venegas (2004).

Una gestión por competencias es requerida para enfrentar confiadamente el cambio, pues hace del aprendizaje una práctica continua donde la gente aprende también a adaptarse al cambio. Por esto, es que en un centro educativo inteligente todos los miembros de la comunidad educativa reaccionan con más agilidad ante los cambios porque saben preverlos (no predecirlos) y así, a su vez, generan los cambios que desean, ya que las relaciones son más importantes que las cosas y la totalidad es más importante que las partes (Martín, 2001). 
Estos elementos hacen que la gestión de la educación por competencias se aprenda, haya motivación y exista una clara y definida conceptualización humana en el servicio educativo. Las interacciones y el clima organizacional referidos fomentan y desarrollan proyectos y sueños en un esfuerzo común, con participación de todos, por lo que se integra el desarrollo óptimo de los planes de estudio, la extensión a la comunidad y la investigación como parte de su misión (OEA-MEP, 2006).

Todo esto es posible porque en un centro educativo inteligente se aplica a plenitud los procesos de gestión por competencias, desde todas sus instancias, incluyendo, desde luego, los diversos proyectos y actividades espontáneas que desarrollan en él. Se trata, pues, de un centro educativo renovado, joven, ágil y flexible, atento a la lectura de los nuevos tiempos, donde profesores, estudiantes y personal no docente, y todos los demás miembros de la comunidad educativa, se identifican también con un espíritu y ambiente de renovación, por lo que están dispuestos a asumir retos (Venegas, 2005).

Surgen así, centros educativos con identidad, que cuidan de su imagen porque son conscientes y han participado activamente en lo que han llegado a ser. Por tanto, se racionaliza y optimiza el uso de los recursos, se trabaja en procesos de autoevaluación, de rediseño curricular y, como perspectiva general, en la planificación participativa constante, ejecución permanente y continua autoevaluación de un plan de desarrollo que ha surgido de sus propias raíces, bajo la guía de lineamientos y políticas generales del Ministerio de Educación que, sin duda alguna, para estar acordes con la época, deben estar fundamentadas en criterios de desconcentración (Venegas, 2009).

Las organizaciones inteligentes que pretendan administrar, manejar y liderar el cambio continuo, la creatividad, la innovación, el aprendizaje, la productividad, la rapidez y la efectividad de todas sus acciones internas y externas, convirtiéndolas en generadoras constantes de valor agregado y satisfacción completa de sus usuarios, deben adecuar velozmente sus estructuras a las nuevas exigencias competitivas (Bolívar, 2000). Esto es fundamental para una adecuada gestión de la educación por competencias. 
Con ello, deben minimizarse los procedimientos burocráticos y darse una auténtica desconcentración, de tal manera que las decisiones se tomen en su propio ámbito de acción, por parte y responsabilidad de los mismos actores.

En este contexto, todos contribuyen a formar la cultura de un centro educativo. No obstante, la dirección tiene una responsabilidad especial, pues ejerce tanta influencia que cualquiera de sus actos tiene gran repercusión en el campo de la organización. Su desempeño, sus conversaciones, sus actos, las decisiones que toman, denotan los valores que trasmiten a la organización. Como expresa Glatter (referido por Venegas, 1995, “...la calidad de la dirección tiene una influencia omnipresente, para bien o para mal” (p.35). Por ello, es que un centro educativo inteligente que realiza sus acciones mediante una gestión por competencias no puede existir sin el compromiso y liderazgo de sus directivos.

Los elementos mencionados hacen que la gestión de la educación, en este inicio del siglo XXI, sea concebida como un nuevo paradigma que incluya, entre otros, los siguientes cambios:

- De una gestión verticalista, con estructuras organizativas jerárquicas, a una gestión de base y participativa

- De una gestión para los otros, a una gestión con los actores

- De una gestión con énfasis en la responsabilidad individual, a una gestión sustentada en la responsabilidad del trabajo en equipo

- De una gestión fundamentada en paradigmas lineales, a una gestión basada en paradigmas no lineales

- De una gestión centrada solo en el producto, a una gestión integral basada en procesos, productos y personas

- De una gestión programática, a una gestión contingencial

- De una gestión centralizada con énfasis en las jefaturas, a una gestión descentralizada y desconcentrada con énfasis en la demanda y necesidades de la comunidad educativa 
- De una cultura reactiva y local, a una cultura organizacional proactiva e internacional

- De una gestión administrativista, a una planificación innovadora

- De una gestión autoritaria y tecnocrática, a una gestión participativa y democrática

- De una gestión normativa, a una gestión situacional

- De una gestión convencional, a una gestión estratégica

- De una gestión con énfasis en el control y lo técnico, a una gestión con énfasis en el compromiso con metas y valores

- De una gestión sustentada en el status quo, a una gestión sustentada en el cambio

- De una gestión que fomenta el control del poder, a una gestión que fomenta la lucha por el poder

- De una planificación basada en la racionalización, a una planificación basada en la innovación

- De la capacitación técnica del personal durante ciertos períodos, a una cultura de aprendizaje continuo

- De una gestión orientada solo hacia la educación formal, a una gestión que también incluye, como factor indispensable, la educación no formal e informal. (Venegas, 2004)

En su carácter de diversidad y multiculturalidad, de acuerdo con los referentes precedentes, se mencionan los siguientes propósitos de las competencias en la gestión de la educación, según Sagastizabal, Perlo, Pivetta, y San Martín (2006):

- Procurar educación integral del individuo para facilitar su inserción en la época actual

- Promover una formación de calidad que permita la solución de problemas reales 
- Articular las necesidades de aprendizaje del individuo con las necesidades del mundo de trabajo

- Integrar teoría y práctica, trabajo manual y trabajo intelectual

- Promover la educación holística

- Promover cambios en lo que los individuos saben y en lo que pueden hacer con lo que saben

- Promover la autonomía del individuo

- Capacitación continua y alterna

- Programas de estudio más flexibles y diversificados

- Contenidos con referencia a las condiciones que opera en la realidad

- Contenidos relevantes y significativos

- Elementos de la competencia: saberes teóricos, práctico/técnico, metodológicos y sociales

- Atributos de la competencia: habilidades, conocimientos, aptitudes, actitudes y valores

- Plan de estudios debe integrar teoría y práctica

A partir de los propósitos de las competencias en la gestión educativa, surge una nueva imagen del profesional en administración de la educación, acorde con las necesidades del siglo XXI, ahora concebido como gestor de la educación, quien requiere aplicar los siguientes principios de las competencias en su ejercicio profesional (Venegas, 2008):

- Transferibilidad: El administrador de la educación debe tener la capacidad de resolver diferentes situaciones en contextos diferentes de manera creativa.

- Multireferencialidad: El administrador de la educación debe estar preparado para aplicar sus conocimientos en diversos contextos. Se reduce la brecha entre lo que se enseña (y se aprende) y las situaciones reales en diferentes contextos. 
- Formación en alternancia: El administrador de la educación es flexible y abierto en su desempeño profesional. Se deja atrás los conceptos tradicionales de la educación (rigidez, control); se asumen programas y proyectos flexibles, abiertos, se combina aprendizaje con la realidad.

- Emergencia de nuevas competencias: Apertura y flexibilidad según cambios del entorno; se dejan atrás las competencias aprendidas que ya no son útiles para adquirir nuevas competencias. El administrador de la educación debe estar abierto al aprendizaje.

- Aprendizaje por disfunciones: El administrador de la educación enfrenta la incertidumbre mediante la puesta en práctica de habilidades creativas y de reflexión para enfrentar situaciones inesperadas.

Retomando los elementos anteriores, en el modelo de educación por competencias, profesionales en la esfera de la administración de la educación, destacan los siguientes enfoques:

a) Definición de tres niveles o dimensiones:

- Competencias básicas: Capacidades intelectuales indispensables para el aprendizaje y desempeño práctico de la administración de la educación. Aquí se ubican las competencias cognitivas, técnicas y metodológicas.

- Competencias genéricas: Base común del ámbito de competencia de la administración de la educación en relación con otras profesiones. Se refieren a todas aquellas situaciones que requieren respuestas concretas para su gestión.

- Competencias específicas: Base particular del ejercicio de la administración de la educación, estrechamente vinculadas a condiciones específicas de ejecución (Instituto Nacional de Formación Técnico Profesional [INFOTEP], 1999). 
b) Factores constantes que están presentes en toda competencia asociada a la administración de procesos educativos:

- Factor cognitivo: conocimientos, habilidades, actitudes, creencias en este ámbito.

- Factor afectivo: sistema de valores, intereses, motivaciones, actitudes que se poseen para realizar las actividades inherentes al campo de la administración de la educación.

- Factor organizacional: relaciones entre el administrador de la educación y el ambiente (Instituto Nacional de Formación Técnico Profesional, 1999).

c) Acepciones complementarias de las competencias en el contexto de la administración de la educación:

- Capacidad: aptitud del administrador de la educación para desarrollar sus deberes y obligaciones.

- Competitividad: capacidad del administrador de la educación para enfrentarse con éxito a nuevos aprendizajes, situaciones o problemas, haciendo valer sus pensamientos y actuaciones.

- Incumbencia: es la obligación que tiene el administrador de la educación de hacer valer su profesión en relación con su realidad y entorno (Instituto Nacional de Formación Técnico Profesional, 1999).

De ahí, que desde el enfoque por competencias, los saberes deben ser:

- Saberes prácticos: Incluyen los atributos de la competencia, saberes técnicos y saberes metodológicos para el ejercicio profesional de la administración de la educación. 
- Saberes teóricos: Conocimientos teóricos requeridos, propios y de disciplinas afines, para el ejercicio de la administración de la educación.

- Saberes valorativos: Querer hacer, predisposición y motivación hacia el autoaprendizaje; saber convivir, capacidad para establecer y desarrollar relaciones sociales. Conjunto de actitudes requeridas para el desempeño del profesional en administración de la educación.

\subsection{Taxonomía de competencias para su interpretación en administración de la educación}

Los elementos precedentes facilitan, a manera de ejemplo, presentar la siguiente clasificación de competencias con el desglose correspondiente según diversas categorías:

- Competencias técnicas, específicas o laborales: Conocimientos, habilidades y actitudes distintivos de las personas de desempeño excepcional en el ámbito de administración de la educación.

- Competencias gerenciales o genéricas: Comportamientos observables y habituales de las personas de desempeño excepcional que les permiten desarrollar su rol de gerencia y ejercer adecuado liderazgo con las personas en el ejercicio de la administración de la educación. Estas se identifican de la siguiente manera, de acuerdo con Pérez (1999):

Tabla 1

Clasificación de las competencias gerenciales

\begin{tabular}{|l|l|}
\hline Competencias & Descripción \\
\hline Competencias estratégicas & $\begin{array}{l}\text { Asociadas con el desarrollo, implementación y } \\
\text { resultados derivados de la estrategia en la } \\
\text { administración de la educación. } \\
\text {-Visión de la organización }\end{array}$ \\
Análisis FODA para construir una visión clara del
\end{tabular}




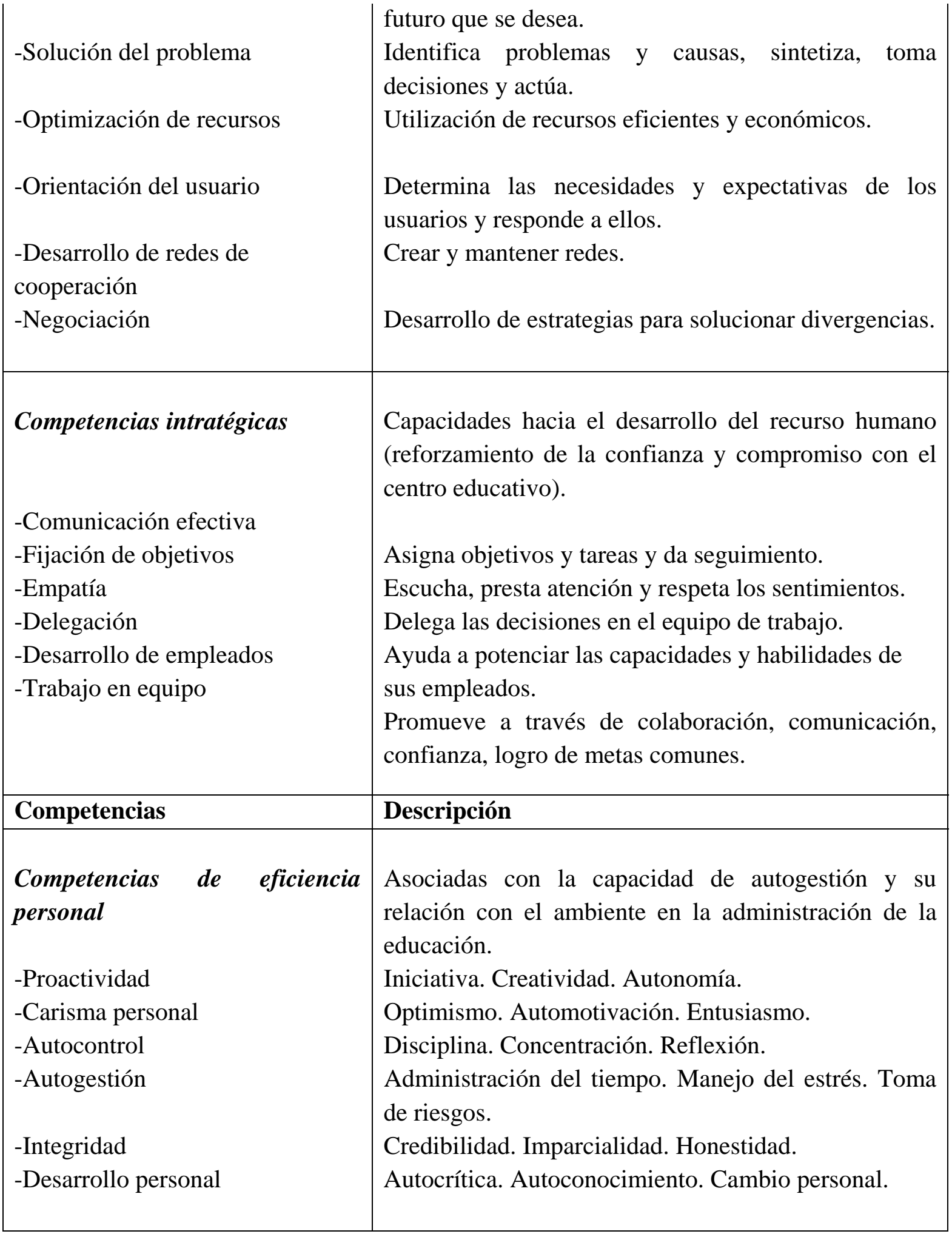


Fuente: Interpretación del autor para efectos de este artículo, de acuerdo con el material bibliográfico utilizado.

Lo anterior conduce a arribar al campo específico de la administración de la educación, en materia de una gestión por competencias, lo que permite un acercamiento a una visión de competencias en ámbito de aprendizaje.

\section{Ideas para un perfil de gestor en administración de la educación}

De acuerdo con Luque (referida por Romero, 2009), la gestión educativa permite y posibilita:

a) Captar la realidad institucional de forma global y en el marco de su propio contexto, de manera democrática y participativa pues comprende al conjunto de los integrantes de la comunidad educativa.

b) Establecer un plan de acción compuesto por acciones sistemáticas, coordinadas e integradas lo que facilita ver como un todo las dimensiones pedagógico-curricular, administrativa, organizativa y comunitaria.

c) Reconocer que la misión educativa de la escuela está focalizada en sus destinatarios y en el logro de más y mejores aprendizajes para ellos.

d) Alcanzar como finalidad el logro de la calidad de la educación con equidad.

e) Analizar las prácticas educativas y generar cambios en la cultura institucional.

Así concebido, la administración de la educación en el contexto de la gestión de la educación basada en competencias, está directamente relacionada con el mejoramiento de la calidad de la educación, mediante mecanismos de participación de todos los miembros de la comunidad educativa, donde los estudiantes constituyen el centro de atención y hacia ellos se dirigen, primordialmente, todas las acciones de la gestión educativa. 
Gestión educativa y cambio en la cultura de centros educativos son sinónimos en el quehacer de la administración de la educación en centros donde se lleven a cabo procesos de enseñanza y aprendizaje (Romero, 2009).

De esta manera, se presentan como referencia de un perfil básico para el administrador de la educación, en términos de una gestión educativa basada en competencias, entre otros, los siguientes elementos que deben caracterizar a este profesional, al menos en las primeras décadas del siglo XXI (Venegas, 2011):

- Saber adaptarse a los cambios y situaciones ambiguas

- Pensar estratégicamente

- Tomar decisiones correctas en situaciones de mucha presión

- Aprender y dominar nuevas tecnologías

- Administrar equipos de trabajo, delegar y ampliar oportunidades

- Favorecer un clima propicio: ampliar desafíos y oportunidades que favorezcan el desarrollo de equipos

- Orientado hacia equipos de trabajo

- Construir equipo de talentos mediante el desarrollo del potencial humano

- Fomentar buenas relaciones: negociación, colaboración, compartir

- Sensibilidad: interés por los demás y tomar decisiones al respecto

- Equilibrio: Establecer prioridades en vida personal y profesional

- Autoconocimiento

- Liderazgo

\subsection{Competencias que debe poseer el profesional en administración de la educación como gestor}

Los elementos previos requieren ser interpretados dentro del contexto de las competencias. En este sentido, el profesional en el campo educativo y en el ejercicio de 
administración de la educación, en particular, debe poseer, entre otras, las siguientes competencias para poder llevar a cabo su papel en un entorno de gestión educativa:

- Competencia conceptual: Entender los fundamentos teóricos de la administración de la educación; todo profesional debe dominar suficientemente los conceptos teóricos que sustentan su profesión.

- Competencia técnica: Habilidad para desempeñar las tareas requeridas en el ámbito de acción de la administración de la educación: aplicar en la práctica, de manera adecuada, lo aprendido.

- Competencia de contexto: Entender el contexto social en el cual se practica la administración de la educción

- Competencia de comunicación: Habilidad para comunicarse en forma oral y escrita, de manera eficaz.

- Competencia de integración: habilidad para combinar las destrezas teóricas y técnicas, en la práctica profesional del administrador de la educación.

- Competencia de adaptación: Habilidad para anticiparse y adaptarse a los cambios, especialmente los cambios tecnológicos. (OEA-MEP, 2006).

La época que se vive obliga a un cambio de paradigma en la formación de administradores de la educación, dirigido a la gestión de la educación mediante un enfoque por competencias. Esto orienta hacia el análisis, reflexión y desarrollo de competencias en diversas áreas. Cada una de ellas debe estar identificada en aspectos como: conceptos, subáreas, logros y niveles de competencias y subcompetencias, así como estrategias de desarrollo de la competencia.

Entre las áreas de gestión y competencias sustantivas para ser incorporadas en la formación y actualización de administradores de la educación, se desatacan las siguientes, como aporte principal del desarrollo de este artículo:

- Gestión de liderazgo 
- Gestión de trabajo en equipo

- Gestión en toma de decisiones

- Gestión curricular

- Gestión de calidad

- Gestión en resolución de conflictos

- Gestión de recursos físicos y financieros

- Gestión de recursos humanos

- Gestión política

- Otros componentes de gestión requeridos para el logro de los propósitos educativos en la administración de la educación.

El cambio que se requiere necesita de una actitud proactiva en el enfoque de gestión de la educación basada en competencias, lo que lleva a un perfil del administrador de la educación acorde con las necesidades reales, propias del momento. Se reitera que los diversos elementos de esta nueva visión son, como mínimo, los que se han planteado en este artículo. Se espera el aporte de profesionales interesados en el campo para ir definiendo, entre todos, este perfil en el contexto de la sociedad del conocimiento y la información.

\section{REFERENCIAS BIBLIOGRÁFICAS.}

Alles, M.A. (2000). Dirección estratégica de recursos humanos: gestión por competencias. Buenos Aires: Granica.

Alles, M.A. (2006). Selección por competencias. Buenos Aires: Ediciones Granica.

Antúnez, S. (2000). Claves para la organización de centros escolares: hacia una gestión participativa y autónoma. Barcelona: Ed. Horsori.

Argüelles, A. (comp.). (1996). Competencia laboral y educación basada en normas de competencia. México: Limusa. 
Arráiz, J. I. (mayo, 2000). Retribución y competencia: ¿cómo garantizar su éxito? Revista Capital humano, 13 (133), 6-8. España: Editorial CISS.

Betancourt, J. R. (2002). Gestión estratégica: navegando hacia el cuarto paradigma. Venezuela: TG Red 2000 Ediciones.

Bolívar, A. (2000). Los centros educativos como organizaciones que aprenden: promesa y realidades. Madrid: La Muralla.

Casassus, J. (2000). Problemas de la gestión educativa en América Latina. La tensión entre los paradigmas de tipo A y el tipo B. París: UNESCO.

Centro Interamericano de Investigación y Documentación sobre Formación Profesional. (2000) ¿Cómo surgió el enfoque de formación basado en competencia laboral? Montevideo, Uruguay: OIT/Cinterfor.

Centro Interamericano de Investigación y Documentación sobre Formación Profesional. (2008). La formación profesional y la productividad. Montevideo, Uruguay: OIT/Cinterfor.

Delors, J., Mufti, I., Amagi, I., Carneiro, R., Chung, F., Geremek, B. y Nanzhao, Z. (1996). La Educación Encierra un Tesoro. Informe a la UNESCO de la Comisión Internacional sobre la Educación para el siglo XXI (Compendio). Francia: Santillana Ediciones UNESCO.

Elizondo C., M. y Mora G., R. (2011). Competencias del educador costarricense en el siglo XXI: manual para contratación y evaluación de educadores de III Ciclo y Educación Diversificada. Informe Taller de Práctica Administrativa Maestría en Ciencias de la Educación con énfasis en Administración de la Educación. Universidad Católica de Costa Rica. San José, Costa Rica.

Instituto Nacional de Formación Técnico Profesional [INFOTEP]. (1999). Manual de procedimiento metodológico para el desarrollo y normalización de competencias laborales. Santo Domingo, República Dominicana: INFOTEP.

Lavín, S. (1998). Gestión integral y gestión participativa: dos orientaciones para una gestión eficaz. Santiago, Chile: IIPE

Luque, M. (2003). Gestión Educativa: Un camino para mejorar la calidad de nuestras escuelas. Colección: Cuadernos para pensar, hacer y vivir la escuela. Ministerio de Educación. Córdoba, Argentina. 
Martín F., E. (2001). Gestión de instituciones educativas inteligentes. Un manual para cualquier tipo de organización. Madrid: Mc Graw Hill.

Ministerio de Educación Pública [MEP]. PROCAE. (1992). La Gestión en la educación. San José, Costa Rica: Departamento de Publicaciones MEP.

Organización Estados Americanos y Ministerio de Educación Pública [OEA-MEP]. (2006). Indicadores para una buena gestión escolar en un modelo educativo basado en competencias. Proyecto Hemisférico Gestión y certificación escolar para la formación y acreditación de competencias laborales y claves en el II nivel de la educación secundaria. San José, Costa Rica: OEA-MEP.

Páez C. Y. (octubre, 2009). Confucio una moral de la cortesía, un saber de la rectitud. Revista Konvergencias. Filosofía y culturas en diálogo, 7 (21). Recuperado de http://www.konvergencias.net/paezcasadiegos257.pdf

Peñate, M. y Luzardo, R. (1995). Administración de instituciones educativas desde la perspectiva del PEI. Colombia: Cooperativa Editorial Magisterio.

Pérez L., A. (abril/mayo, 1999). Evaluación y desarrollo de competencias directivas. Harvard Deusto Business Review, 89. Recuperado de: kino.iteso.mx/luisg/EVALUACION\%20Y\%20DESARROLLO\%20DE\%20LAS\%2 0COMPETENCIAS\%20DIRECTIVAS.doc

Pozner, P. (2000). Competencias para la profesionalización de la gestión educativa. Argentina: IIPE.

Pozner, P. (2000). Diez módulos destinados a los responsables de los procesos de transformación educativa. Ministerio de Educación. Buenos Aires: IIPE.

Pozner, P. (1995). El directivo como gestor de aprendizajes escolares. Buenos Aires: Aique.

Romero, C. (2004). La escuela media en la sociedad del conocimiento: Ideas y herramientas para la gestión educativa. Argentina: Noveduc.

Romero, C. (2009). Hacer de una escuela, una buena escuela: evaluación y mejora de la gestión escolar. Buenos Aires: Aique.

Sagastizabal, M. A.; Perlo, C.; Pivetta, V.; San Martín, P. (2006). Enseñar y aprende en contextos complejos: multiculturalidad, diversidad y fragmentación. Buenos Aires: Noveduc. 
Sander, B. (1996). Gestión educativa y calidad de vida. Implicaciones para América Latina. Revista La Educación, 38 (18). Washington, DC. : OEA.

Sander, B. (1996). Historia del pensamiento administrativo en la educación latinoamericana. Gestión educativa en América Latina: construcción y reconstrucción del conocimiento. Buenos Aires: Editorial Troquel.

Senge, M. (1998). La quinta disciplina en la práctica: estrategias y herramientas para construir la organización abierta al aprendizaje. Barcelona: Ediciones Granica.

UNESCO. (2004). Management in Latin America and the Caribbean, are we on the right track? Recuperado de http://portal.unesco.org/geography/en/ev.phpURL_ID=8299\&URL_DO=DO_TOPIC\&URL_SECTION=201.html

Venegas J., P. (1995). Innovación y cambio en educación: el director y el docente como partícipes medulares. San José, Costa Rica: Oficina de Publicaciones de la Universidad de Costa Rica.

Venegas J., P. (2003). Administración de la educación y planificación educativa en tiempos de cambio y aprendizaje permanente: el P.E.C.E. como funcion vital del centro educativo. III Encuentro Nacional de Experiencias Exitosas en Administración Educativa. Universidad Latina de Costa Rica. San José, Costa Rica.

Venegas J., P. (2004). Planificación educativa: bases metodológicas para su desarrollo en el siglo XXI. San José, Costa Rica: EUNED.

Venegas J., P. (2008). Gestión educativa basada en competencias. Congreso educativo organizado por el Colegio Profesional de Licenciados y Profesores en Letras, Filosofía, Ciencias y Artes (COLYPRO). San José, Costa Rica.

Venegas J., P. (2009). El administrador educativo que se necesita para el siglo XXI. Primer Congreso para Administradores Educativos ADEM. San José, Costa Rica.

Venegas J., P. (2011). La educación en un contexto de calidad: enfoques siglo XXI. Lección Inaugural Curso Lectivo 2011. Dirección Regional de Turrialba. Cartago, Costa Rica. 